under review as of Aug 18, 2021

\title{
Weak nuclear spin singlet relaxation mechanisms revealed by experiment and computation
}

\author{
Boris Kharkov, ${ }^{1}$ Xueyou Duan, ${ }^{2}$ Jyrki Rantaharju, ${ }^{3}$ Mohamed Sabba, ${ }^{3}$ \\ Malcolm H. Levitt, ${ }^{3}$ James W. Canary ${ }^{2}$ and Alexej Jerschow ${ }^{* 2}$ \\ ${ }^{1}$ Laboratory of Biomolecular NMR, St. Petersburg \\ State University, St. Petersburg 199034, Russia \\ ${ }^{2}$ Department of Chemistry, New York University, New York, NY 10003 \\ ${ }^{3}$ School of Chemistry, University of Southampton, Southampton, UK
}




\begin{abstract}
Nuclear spin singlet states are often found to allow long lived storage of nuclear magnetization, which can form the basis of novel applications in spectroscopy, imaging, and in studies of dynamic processes. Precisely how long such polarization remains intact, and which factors affect its lifetime is often difficult to determine and predict. We present a combined experimental/computational study to demonstrate that molecular dynamics simulations and ab initio calculations can be used to fully account for the experimentally observed singlet lifetimes in an organic molecule in solution. The correspondence between experiment and simulations is achieved without adjustable parameters. These studies highlight the importance of considering unusual and difficult-to-control mechanisms, such as dipolar couplings to low-gamma solvent nuclei, and to residual paramagnetic species, which often can represent lifetime limiting factors. These results also point to the power of molecular dynamics simulations to provide insights into little-known NMR relaxation mechanisms.
\end{abstract}

\title{
MAIN TEXT
}

The ability of nuclear spin singlet order (SO) to exhibit lifetimes much longer than spin lattice relaxation times has motivated the investigation into the use of such states as information or polarization storage vehicles [1-3]. Potential applications include imaging [4], the study of slow kinetic or dynamic processes $[5,6]$, or the study of weak relaxation mechanisms $[7,8]$.

The mechanisms that ultimately lead to the decay of SO are often difficult to identify and quantify. Molecular dynamics (MD) and ab initio calculations (including ab initio MD) have been used for the calculation, prediction, and analysis of NMR processes [9-15], but this approach has not been extended to nuclear spin singlet states, with the exception of the work of Håkansson [16]. That work presented a unique approach to use a fitting algorithm to interpolate between different MD snapshots for which ab initio quantities were calculated. In that work, the spin rotation, chemical shift anisotropy and intramolecular dipolar mechanisms were calculated, but intermolecular mechanisms were not included. As shown below, intermolecular mechanisms represent major components of the relaxation rate constants, and can represent lifetime limiting factors.

Here, we report on nuclear spin singlet lifetime measurements in an organic molecule at different temperatures, and calculate the underlying singlet relaxation mechanisms using 
MD simulations and ab initio calculations. MD simulations are used, in particular, to derive quantities for the intermolecular dipolar coupling mechanism to nuclei in solvent molecules $\left({ }^{2} \mathrm{H},{ }^{35} \mathrm{Cl},{ }^{37} \mathrm{Cl}\right)$. Another significant effect is given by chemical shift anisotropy (CSA), which is treated using a combination of ab initio calculations and MD simulations.

In addition, the paramagnetic mechanism due to dissolved oxygen is modeled as a dipolar mechanism to a spin-1 object with an electronic relaxation time obtained from the literature. Paramagnetic relaxation is potentially a very complex subject, and we highlight below the simplifying assumptions and justifications used in order to make the calculation of this effect from MD trajectories tractable. The paramagnetic effect is further treated in a selfconsistent manner, by extracting a scaling factor from its contribution to solvent spin-lattice relaxation.

Overall, the calculation of all these mechanisms accounts for the measured rate constants over the temperature range considered. This work points, in particular, to the fact that dipolar interactions with low-gamma nuclei, such as ${ }^{2} \mathrm{H},{ }^{35} \mathrm{Cl}$, and ${ }^{37} \mathrm{Cl}$ of the solvent could ultimately be lifetime limiting factors in the quest for the longest nuclear spin singlet lifetimes in solution.

All experiments were performed on a degassed $5.4 \mathrm{mM}$ solution of ethyl- $\mathrm{d}_{5}$ propyl- $\mathrm{d}_{7}$ maleate $(\mathrm{EPM})$ in $\mathrm{CDCl}_{3}$. $\mathrm{SO}$ was established between the two vinylene protons. The synthesis and sample preparation were performed in analogy to the previously described procedure $[17,18]$, and is provided in detail in the Supplemental Material document.

The measurements of the relaxation of the ${ }^{1} \mathrm{H}$ spins of residual $\mathrm{CHCl}_{3}$ in the same sample $(<0.2 \%)$ provided a means of benchmarking the calculation approach and extracting the relative weight of the paramagnetic relaxation effects.

Figure 1 shows the measured chloroform ${ }^{1} \mathrm{H} R_{1}$ rate constants in the degassed solution, as well as the calculated rate constants for the main relaxation mechanisms. The relaxation mechanisms of chloroform have been studied previously [19, 20], and the main contributions have been determined as arising from the intra- and intermolecular dipolar coupling (to ${ }^{2} \mathrm{H},{ }^{35} \mathrm{Cl}$, and ${ }^{37} \mathrm{Cl}$ ), as well as the spin-rotation interaction. We believe, however, that the contribution of oxygen has previously not been determined. It is well-known that in a nondegassed sample, the paramagnetic mechanism accounts for the majority of the effect. In the degassed sample used in this study, the oxygen concentration is not known, but since one can calculate the dipolar coupling and the spin-rotation mechanisms from MD and ab 
initio simulations, one can assume that the remaining contribution must be due to oxygen. Figure 1a shows the contributions of the different dipolar mechanisms. It is observed here that the dipolar mechanisms due to ${ }^{35} \mathrm{Cl}$ and ${ }^{37} \mathrm{Cl}$ are much stronger that those due to ${ }^{2} \mathrm{H}$. The reason is that there is no intramolecular mechanism due to ${ }^{2} \mathrm{H}$, but more importantly, there are three times more chlorine spins than there are ${ }^{2} \mathrm{H}$ spins, and their spin value is larger as well. Overall, this combination of factors explains the large contribution from chlorine spins as shown in Fig. 1a.

MD calculation details are provided in the Supplemental Material document. The dipolar contribution was calculated for ${ }^{1} \mathrm{H}-{ }^{2} \mathrm{H},{ }^{1} \mathrm{H}-{ }^{35} \mathrm{Cl}$, and ${ }^{1} \mathrm{H}-{ }^{37} \mathrm{Cl}$ spin pairs using the following expression:[21]

$$
R_{1}=\left(\frac{\mu_{0}}{4 \pi} \hbar \gamma_{I} \gamma_{S}\right)^{2} \frac{4}{3} S(S+1) \frac{1}{10}\left(J\left(\left|\omega_{I}-\omega_{S}\right|\right)+3 J\left(\omega_{I}\right)+6 J\left(\omega_{I}+\omega_{S}\right)\right),
$$

where $\mu_{0}$ is the permeability of vacuum, $I$ and $S$ are the spin values, $\gamma_{I, S}$ are the gyromagnetic ratios, the index $I$ refers to the spin species for which the relaxation is being determined (here ${ }^{1} \mathrm{H}$ ), and $S$ to the spin whose dipolar coupling to spin $I$ is the cause of the mechanism. $\omega_{I, S}$ are the respective (angular) Larmor frequencies. The factor $\frac{4}{3}$ arises from the $I(I+1)$ factor for spin $1 / 2$, and disappears when combined with $S(S+1)$ if a coupling between two spin-1/2 nuclei is considered.

$J(\omega)$ is the spectral density function given by the Fourier transformation of the secondrank corrleation function $C_{2}(t)$,

$$
J(\omega)=\int_{0}^{\infty} C_{2}(t) \exp (-i \omega t) d t
$$

The function $C_{2}(t)$ is calculated from the MD trajectories using

$$
C_{2}(t)=5 \overline{a_{2}\left(t^{\prime}\right)^{*} a_{2}\left(t+t^{\prime}\right)}
$$

where the average is performed over $t^{\prime}$, with

$$
a_{2}(t)=P_{2}\left(\cos \left(\theta_{I S}\right)\right) / r_{I S}^{3}
$$

$\theta_{I S}$ is the angle that the inter-spin vector makes with the $z$ axis, $P_{2}$ is the second-order Legendre polynomial, and $r_{I S}$ is the internuclear distance. The factor 5 in Eq. (4) arises from the normalization of the $P_{2}$ function when performing the spherical integral.

In this study, the largest correlation time was determined for the ${ }^{1} \mathrm{H}-{ }^{35,37} \mathrm{Cl}$ coupling for the lowest examined temperature $(220 \mathrm{~K})$. For this situation, we show the correlation 
function in Fig. S2. This curve can be fit very well with a biexponential function to give the two correlation times, 77.3ps and 11.1ps. From these two correlation times one may determine the deviation from the fast motion assumption as being at the levels of $9.6 \cdot 10^{-4}$ and $1.96 \cdot 10^{-5}$, both of which can be neglected. Therefore, the fast motion regime can be applied to simplify the expressions for the system considered. This approach also avoids the use of any particular correlation function model (e.g. expoential decay), and one can hence make the approximation

$$
J(\omega) \approx J(0)=\int_{0}^{\infty} C(t) d t .
$$

The integration was performed to an upper limit of 800 ps in this study.

The same equations and procedures were used for both intra- and intermolecular processes, except for the following additional considerations for the latter: We analyzed the convergence behavior of the relaxation expressions when choosing particular cutoff distances for choosing intermolecular coupling partners. As seen in Fig. S3, a cutoff distance of $20 \AA$ represents well-converged results, and hence this value was chosen for the cutoff distance.
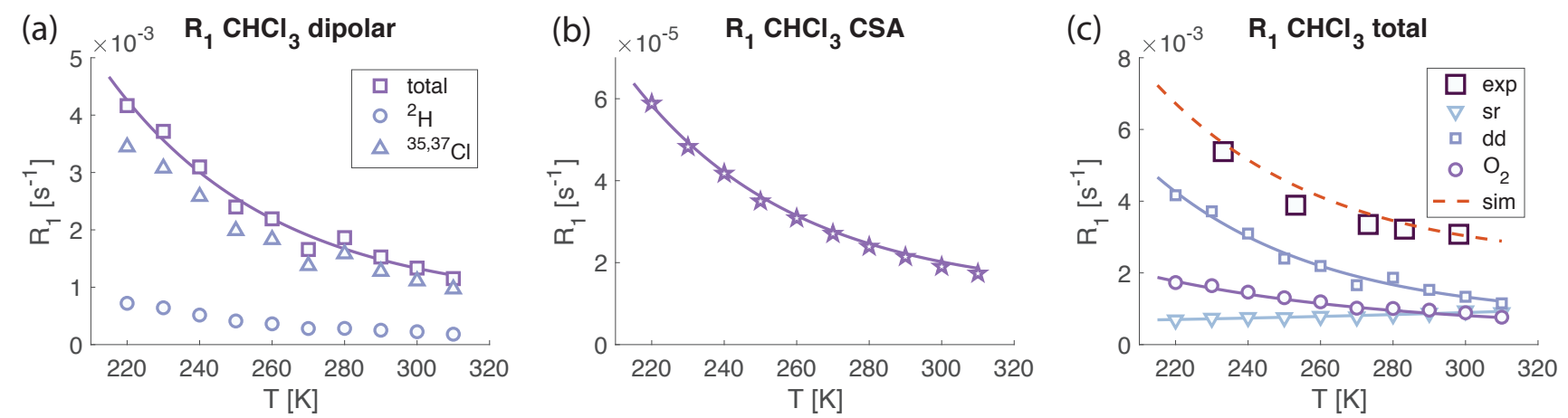

FIG. 1. Measured and calculated ${ }^{1} \mathrm{H} R_{1}$ rate constants for $\mathrm{CHCl}_{3}$ (in $\mathrm{CDCl}_{3}$ ). (a) Components of the dipolar coupling mechanism showing the ${ }^{1} \mathrm{H}-{ }^{2} \mathrm{H}$ and the ${ }^{1} \mathrm{H}_{-}-35,37 \mathrm{Cl}$ contributions. (b) CSA relaxation contribution (only the symmetric component is non-zero for chloroform). (c) Spinrotation ( $\mathrm{sr}$ ), dipolar coupling $(\mathrm{dd})$, paramagnetic $\left(\mathrm{O}_{2}\right)$ contributions, and their sum (red dashed line, labeled 'sim'), along with the experimentally determined values (exp).

Fig.1b shows estimates of the CSA contributions to the $R_{1}$ rate constant for ${ }^{1} \mathrm{H}$ relaxation in $\mathrm{CHCl}_{3}$. The CSA contribution is estimated to be approximately a factor 100 smaller than other contributions), as seen in Fig. 1b. Only the symmetric tensor is non-zero for the ${ }^{1} \mathrm{H}$ chloroform spin and $\left\|\sigma_{s y m}\right\|_{F}$ is found to be approximately $3.05 \mathrm{ppm}$. All CSA tensor 
calculation details are described in the Supplemental Materials document.

In Fig. 1c we show the spin-rotation contribution, the overall dipolar contribution, as well as the paramagnetic relaxation contribution, compared to the experimentally determined values.

The expression for the spin-rotation relaxation can be derived for a spherical top molecule (such as chloroform) using references $[22,23]$ in terms of the principal components of the moments of inertia $\left(I_{\perp}, I_{\|}\right)$and diffusion tensors $\left(D_{\perp}, D_{\|}\right)$as

$$
R_{1}^{s r}=\frac{2}{3 \hbar^{2}}\left(I_{\|}^{2} C_{\|}^{2} \mathrm{D}_{\|}+2 I_{\perp}^{2} C_{\perp}^{2} \mathrm{D}_{\perp}\right) .
$$

A derivation of this expression is given in the Supplemental Material document.

Eq. (6.27) of Kowalewski and Mäler [23] may be adapted to give the following relationship between the diffusion constants and small orientational changes:

$$
\begin{gathered}
D_{\|} \simeq \frac{\delta \theta_{\|}^{2}}{\delta t} \\
D_{\perp} \simeq \frac{\delta \theta_{\perp}^{2}}{\delta t} .
\end{gathered}
$$

Here $\delta \theta_{\|}$and $\delta \theta_{\perp}$ are small rotation angles parallel and perpendicular to the symmetry axis, respectively, accumulated over a small time interval $\delta t$ (which is $0.2 \mathrm{ps}$ in this case).

The spin-rotation tensor components for chloroform are well-known, but can also be straightforwardly calculated. The values obtained using Gaussian16 were $C_{\|} / 2 \pi=100 \mathrm{~Hz}$, and $C_{\perp} / 2 \pi=300 \mathrm{~Hz}$, respectively. Note that some published expressions for spin-rotation relaxation contain errors in the numerical factors[19, 20].

The relaxation of nuclei by the interaction with unpaired electrons is a complex topic $[24,25]$. A detailed treatment of this problem requires good understanding of the electron spin interactions including zero-field splittings. For the sake of tractability, we employed a simplified treatment based on Solomon relaxation of the $\mathrm{O}_{2}$ electron spin pair. The relaxation contribution was determined by calculating

$$
R_{1}^{O_{2}}=R_{1}^{O_{2}, d d}+\frac{1}{T_{1 e}}
$$

where $R_{1}^{O_{2}, d d}$ is the spin-lattice relaxation relaxation rate constant determined using the procedure above for intermolecular dipolar relaxation contributions with $S=1$ for the unpaired electrons of $\mathrm{O}_{2}$, and the gyromagnetic ratio for the electron. The electron relaxation 
time $T_{1 e}$ was recently reported to be $7.5 \mathrm{ps}$ for most organic solvents at room temperature [26], which is the value we used. We note that an increase in $T_{1 e}$ would lead to larger rate constants, and hence a decrease in the determined oxygen concentration. The Supplemental Material document contains an evaluation of its influence (Fig. S4). The scaling factor determined from $R_{1}$ data was subsequently used to scale the computed interaction for the contribution to the SO relaxation rate constant $R_{S}$. As a result, we obtain a self-consistent procedure for determining the paramagnetic relaxation contribution to $R_{S}$.

One may also consider the potential effect of ${ }^{1} \mathrm{H}^{1}{ }^{1} \mathrm{H}$ dipolar coupling due to the fraction of protonated solvent $(<0.2 \%)$. We can estimate the size of this contribution from the ${ }^{1} \mathrm{H}-{ }^{3} \mathrm{H}$ dipolar coupling contribution as follows: The largest $R_{1}$ rate constant due to ${ }^{2} \mathrm{H}$ is $7.2 \times 10^{-4}$ $\mathrm{s}^{-1}$. The factor

$$
c_{H D}=\left(\gamma_{D} / \gamma_{H}\right)^{2} \frac{I_{D}\left(I_{D}+1\right)}{I_{H}\left(I_{H}+1\right)}=0.0628
$$

represents the conversion factor between the relaxation rate constants due to proton and deuterium $\left(I_{D}=2\right.$ and $I_{H}=1 / 2$ are the spin values of ${ }^{2} \mathrm{H}$ and ${ }^{1} \mathrm{H}$, respectively). Therefore, the estimate of the effect of residual ${ }^{1} \mathrm{H}$ on chloroform $R_{1}$ is $7.2 \times 10^{-4} \cdot 0.002 / c_{H D}=$ $2.29 \times 10^{-5} \mathrm{~s}^{-1}$. This rate constant is clearly negligible (more than a factor 100 smaller than the smallest calculated contributions to $R_{1}$ ).

Next, we turn our attention to the measurement and computation of SO relaxation rate constants $R_{S}$ for the vinylene protons in the EPM molecule. To measure the singlet lifetimes we used a M2S pulse sequence with 25-pulse composite 180 pulses [18] and a zero-rank tensor filter [27] (subsequently referred to as T00 filter) as shown in Fig. 2. This method was previously described as a technique for minimizing convection and rf inhomogeneity artifacts, which can appear in experiments with very long singlet lifetimes [27]. The T00 filter removes spin order other than SO up to rank two. Convection was further controlled by restricting the height of the solution in the NMR tube to within the active rf coil volume. [18]. The T00 filter removes spin order other than SO up to rank two.

The Supplemental Material document contains further NMR acquisition and sequence parameters including pulse sequence optimization data (Table S1, in particular), as well as validation computations of vinylene $R_{1}$ rates, which demonstrate the legitimacy of computed rotational correlation times.

Figure 3 compares the experimentally measured $R_{S}$ rate constants for the vinylene protons of EPM along with the computed ones. Fig. 3a shows the breakdown between the different 


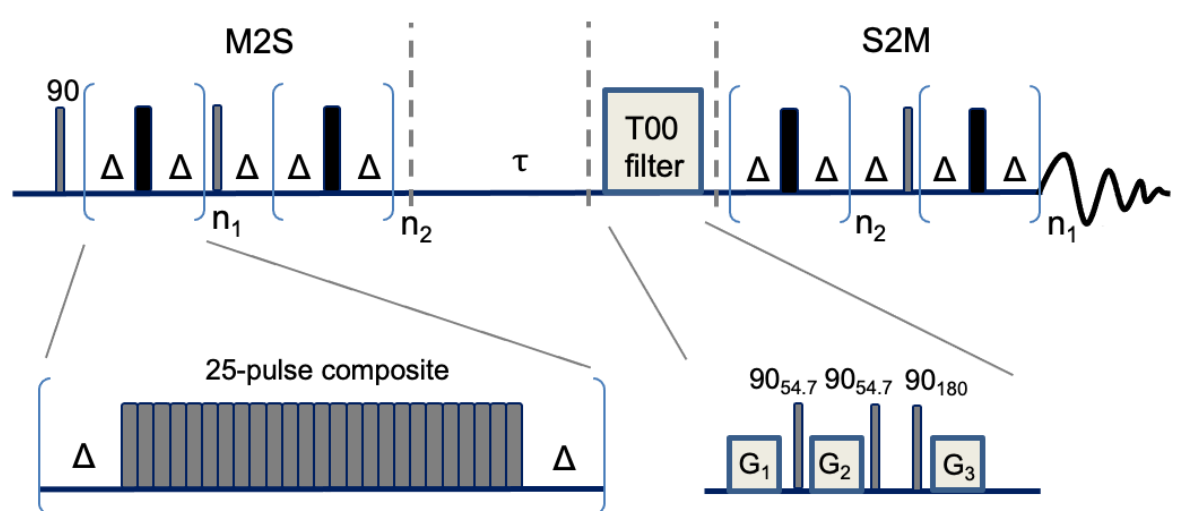

FIG. 2. M2S-S2M sequence with zero-rank (T00) filter and 25-component composite pulses [18].
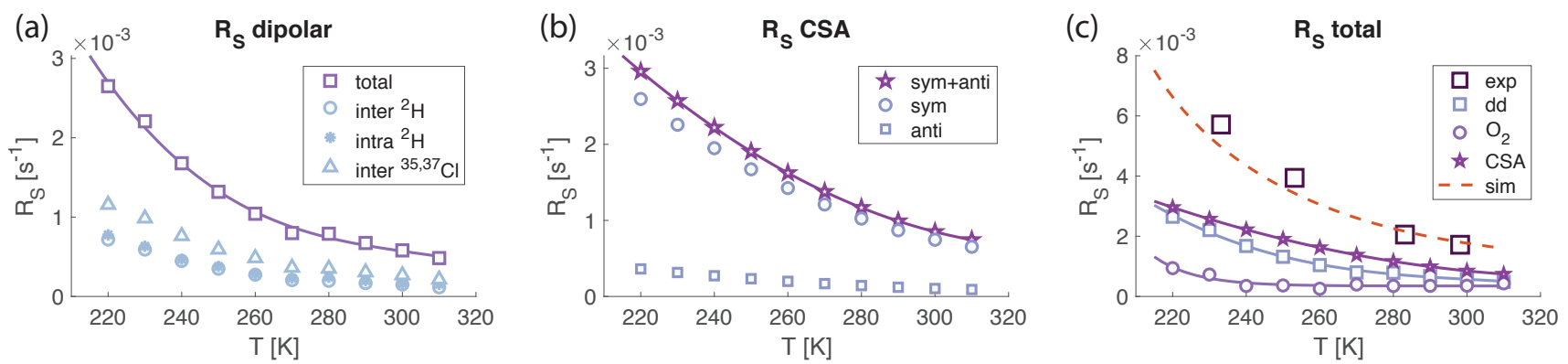

FIG. 3. Measured and calculated $R_{S}$ rate constants for the vinylene protons in $\mathrm{EPM}$ in $\mathrm{CDCl}_{3}$. (a) Components of the dipolar coupling mechanism showing the intra- and intermolecular ${ }^{1} \mathrm{H}-{ }^{2} \mathrm{H}$, and the intermolecular ${ }^{1} \mathrm{H}_{-}{ }^{35,37} \mathrm{Cl}$ contributions. (b) Symmetric (sym) and antisymmetric (anti) CSA relaxation contributions. (c) CSA, dipolar coupling (dd), paramagnetic $\left(\mathrm{O}_{2}\right)$ contributions, and their sum (red dashed line, labeled 'sim'), along with the experimentally determined values $(\exp )$

dipolar coupling mechanisms, including both intra- and intermolecular ones. As for the $R_{1}$ calculation above, it is observed here that the interactions with ${ }^{35} \mathrm{Cl}$, and ${ }^{37} \mathrm{Cl}$ are much stronger overall than those with ${ }^{2} \mathrm{H}$, although the difference is not as large as seen for chloroform $R_{1}$. Both the intra- and intermolecular ${ }^{2} \mathrm{H}$ interactions are of a similar level, approximately at $70 \%$ of the interactions with chlorine spins.

$R_{S}$ rate constants due to dipolar coupling were calculated as follows: Although it is not difficult, in principle, to calculate the exact relaxation rate constant according to equations given by Pileio [28], a more efficient algorithm can be used when the fast motion approximation applies. The justification for the use of the fast motion regime was mentioned above 
already and is corroborated by the data provided in Fig. S2.

SO relaxation rate constants due to dipolar coupling were calculated in this regime by

$$
R_{S}=\frac{4}{3}\left(\frac{\mu_{0}}{4 \pi} \hbar \gamma_{I} \gamma_{S}\right)^{2} S(S+1) J(0)
$$

where the spectral density function was given by Eq. (5), with the correlation function calculated in this case by

$$
C_{2}(t)=C_{S O}(t)=5 \overline{a_{S O}^{*}\left(t^{\prime}+t\right) a_{S O}\left(t^{\prime}\right)}
$$

where

$$
a_{S O}(t)=P_{2}\left(\cos \left(\theta_{I_{1} S}\right)\right) / r_{I_{1} S}^{3}-P_{2}\left(\cos \left(\theta_{I_{2} S}\right)\right) / r_{I_{2} S}^{3}
$$

with the subscripts to $I$ and $r$ indicating for which spin of the two spin- $1 / 2$ the quantities are evaluated. The paramagnetic contribution to $R_{S}$ was calculated using the same expressions as for dipolar coupling, with the substitutions for the spin value $(S=1)$ and the electron gyromagnetic ratio. In addition, a correction for $T_{1 e}$ was performed in analogy to Eq. (9).

Figure $3 \mathrm{~b}$ shows the calculated CSA contribution to $R_{S}$ performed from MD snapshots according to the procedure given in the Supplemental Material document. The CSA difference tensor norms were found to be $\left\|\Delta \sigma_{\text {sym }}\right\|_{F}=4.35 \mathrm{ppm}$ and $\left\|\Delta \sigma_{\text {anti }}\right\|_{F}=0.94 \mathrm{ppm}$. The standard deviations of 0.13 and $0.18 \mathrm{ppm}$, respectively, indicate that the values do not fluctuate significantly, especially for the symmetric component. It is seen that the contribution from the symmetric interaction is significantly stronger than the antisymmetric one.

Figure $3 \mathrm{c}$ shows all the mechanisms (including the paramagnetic interaction due to oxygen) together, as well as their sum. It is seen that the calculated rate constants track the experimentally observed very well, with a very slight underestimation. This result of particular interest, given that no adjustable parameters were used. Figure $3 \mathrm{c}$ also shows that overall, the effect of oxygen is relatively minor, but not negligible. The level of the relaxation mechanism, however, is a testament to the importance of thorough oxygen removal for the measurement of long singlet lifetimes, as an oxygen concentration that would be higher by a factor three would eclipse the dipolar contribution.

As above, one can estimate the potential contribution of residual ${ }^{1} \mathrm{H}$ in deuterated chloroform: The largest $R_{S}$ rate constant due to intermolecular ${ }^{2} \mathrm{H}$ is $7.77 \times 10^{-4} \mathrm{~s}^{-1}$. Therefore, the estimate of the effect of residual solvent ${ }^{1} \mathrm{H}$ on EPM $R_{S}$ is $7.77 \times 10^{-4} \cdot 0.002 \mathrm{~s}^{-1} / c_{H D}=$ 
$2.47 \times 10^{-5} \mathrm{~s}^{-1}$, which is more than a factor 100 smaller than the tinyest dipolar coupling contribution to $R_{S}$.

Furthermore, we consider whether EPM-EPM dipolar interactions could play a role. An estimate for such potential contributions could be obtained as follows: The EPM concentration is $5.4 \mathrm{mM}$. There are two ${ }^{1} \mathrm{H}$ spins in the deuterated EPM molecule that we use. The concentration of neat $\mathrm{CHCl}_{3}$ is $13 \mathrm{M}$. We calculated above the relaxation contributions due to a 0.002 fraction of that amount. Therefore, any dipolar contribution from distant EPM molecules would be scaled by a factor $2 \cdot 0.0054 /(13 \cdot 0.002)=0.415$ from the rate constant considered above. Hence this contribution would therefore be at a level of approximately $1 \times 10^{-5} \mathrm{~s}^{-1}$ for EPM $R_{S}$, which we can also safely neglect.

The contribution to $R_{S}$ from dipolar coupling and the CSA interaction are the largest and are roughly of the same order over the whole temperature range. It is of note that one could remove the CSA interaction by reducing the external field. It is interesting to estimate the limit of singlet lifetimes under such conditions. For the molecule examined here, the strongest contribution would then be given by dipolar coupling, which would produce lifetime limits of $578 \mathrm{~s}$ at $220 \mathrm{~K}$ and $1127 \mathrm{~s}$ at $300 \mathrm{~K}$. It is of note that the biggest component thereof is given by the intermolecular coupling to chlorine spins, something that is rarely being considered. Since singlet NMR typically requires a solvent, one can see such interactions as presenting hard limits to singlet lifetimes. From the effect on ${ }^{1} \mathrm{H}$, one can further deduce expected limits for singlet lifetimes for other nuclear species. For example, the record for organic molecules has been established as over an hour for ${ }^{13} \mathrm{C}$ spin pairs $[3,29]$. Since the dipolar relaxation contribution scales with $\gamma^{2}$, the lifetime limit due to the solvent would become approximately $1.2 \mathrm{~h}$ in a solvent such as chloroform, if we only consider the $\gamma$ factor. The lifetime limit would likely be a little longer because the distance of closest approach would be larger for ${ }^{13} \mathrm{C}$ spins. Nonetheless, this consideration appears to indicate that the lifetimes observed thus far in the literature are already very close to the theoretical limits given by solvent effects. This finding may also be an additional motivation to opt for supercritical $\mathrm{CO}_{2}$ as a solvent [29].

In summary, we discuss here results from an experimental and computational study of different nuclear spin singlet relaxation mechanisms in order to reproduce the temperature behavior of experimentally observed relaxation rate constants of an organic molecule in solution. In particular, it is shown that a combination of intra- and intermolecular dipolar 
coupling relaxation $\left({ }^{1} \mathrm{H}_{-}{ }^{2} \mathrm{H},{ }^{1} \mathrm{H}-{ }^{35,37} \mathrm{Cl}\right.$ ), chemical shift anisotropy (both symmetric and antisymmetric), as well as a relatively minor contribution from paramagnetic relaxation due to residual oxygen can fully account for the observed rate constants of an organic molecule in solution. Self-consistency in the predictions is achieved by examining the solvent relaxation in order to determine the contribution of oxygen to relaxation. This study further identifies potentially hard limits on nuclear spin singlet relaxation rate constants given by solvent interactions (even in deuterated solvents), which may set an upper boundary on singlet lifetimes in solution.

\section{ACKNOWLEDGEMENTS}

We acknowledge funding though an award of the U.S. National Science Foundation, award no. CHE 2108205, and an award by the Heising-Simons Foundation. AJ acknowledges the receipt of a Diamond Jubilee Visiting Fellowship to the University of Southampton. This work was supported in part through the NYU IT High Performance Computing resources, services, and staff expertise (in particular from Dr. Shenglong Wang). B.B. Kharkov was supported by grant 72777155 from St. Petersburg State University awarded to the Laboratory of Biomolecular NMR at SPbSU. This research was further supported by EPSRC-UK (grant numbers EP/P009980/1, EP/T004320/1 and EP/P030491/1), and the European Union's Horizon 2020 research and innovation programme under the Marie Skłodowska-Curie grant agreement No 891400, and the European Research Council (grant 786707-FunMagResBeacons). We acknowledge extensive discussions with Dr. Christian Bengs with regard to nuclear spin singlet relaxation mechanisms.

[1] M. Carravetta, O. G. Johannessen, and M. H. Levitt, Beyond the t1 limit: singlet nuclear spin states in low magnetic fields, Phys Rev Lett 92, 153003 (2004).

[2] M. Carravetta and M. H. Levitt, Long-lived nuclear spin states in high-field solution nmr, J Am Chem Soc 126, 6228 (2004).

[3] G. Stevanato, J. T. Hill-Cousins, P. Hakansson, S. S. Roy, L. J. Brown, R. C. Brown, G. Pileio, and M. H. Levitt, A nuclear singlet lifetime of more than one hour in room-temperature solution, Angew Chem Int Ed Engl 54, 3740 (2015). 
[4] G. Pileio, S. Bowen, C. Laustsen, M. C. Tayler, J. T. Hill-Cousins, L. J. Brown, R. C. Brown, J. H. Ardenkjaer-Larsen, and M. H. Levitt, Recycling and imaging of nuclear singlet hyperpolarization, J Am Chem Soc 135, 5084 (2013).

[5] S. Cavadini, J. Dittmer, S. Antonijevic, and G. Bodenhausen, Slow diffusion by singlet state nmr spectroscopy, J Am Chem Soc 127, 15744 (2005).

[6] R. Sarkar, P. R. Vasos, and G. Bodenhausen, Singlet-state exchange nmr spectroscopy for the study of very slow dynamic processes, J Am Chem Soc 129, 328 (2007).

[7] Y. Zhang, X. Duan, P. C. Soon, V. Sychrovsky, J. W. Canary, and A. Jerschow, Limits in proton nuclear singlet-state lifetimes measured with para-hydrogen-induced polarization, ChemPhysChem 17, 2967 (2016).

[8] S. J. Elliott, L. J. Brown, J.-N. Dumez, and M. H. Levitt, Long-lived nuclear spin states in rapidly rotating CH2D groups, J. Magn. Reson. 272, 87 (2016).

[9] A. Philips and J. Autschbach, Proton NMR relaxation from molecular dynamics: intramolecular and intermolecular contributions in water and acetonitrile, Phys Chem Chem Phys 21, 26621 (2019).

[10] S. Badu, L. Truflandier, and J. Autschbach, Quadrupolar NMR Spin Relaxation Calculated Using Ab Initio Molecular Dynamics: Group 1 and Group 17 Ions in Aqueous Solution, J Chem Theor Comput 9, 4074 (2013).

[11] A. Carof, M. Salanne, T. Charpentier, and B. Rotenberg, Collective water dynamics in the first solvation shell drive the NMR relaxation of aqueous quadrupolar cations, J. Chem. Phys. 145, 124508 (2016).

[12] M. Mohammadi, S. Benders, and A. Jerschow, Nuclear magnetic resonance spin-lattice relaxation of lithium ions in aqueous solution, studied by nmr spectroscopy and molecular dynamics simulations, J Chem Phys 153, 184502 (2020).

[13] R. Brüschweiler, New approaches to the dynamic interpretation and prediction of nmr relaxation data from proteins, Curr. Opinion Struct. Biol. 13, 175 (2003).

[14] M. Odelius, A. Laaksonen, M. H. Levitt, and J. Kowalewski, Intermolecular dipole-dipole relaxation. a molecular dynamics simulation, J. Magn. Reson., Ser. A 105, 289 (1993).

[15] M. Odelius, C. Ribbing, and J. Kowalewski, Spin dynamics under the hamiltonian varying with time in discrete steps: Molecular dynamics-based simulation of electron and nuclear spin relaxation in aqueous nickel (ii), J. Chem. Phys. 104, 3181 (1996). 
[16] P. Håkansson, Prediction of low-field nuclear singlet lifetimes with molecular dynamics and quantum-chemical property surface, Phys Chem Chem Phys , 1 (2017).

[17] G. Stevanato, S. S. Roy, J. Hill-Cousins, I. Kuprov, L. J. Brown, R. C. Brown, G. Pileio, and M. H. Levitt, Long-lived nuclear spin states far from magnetic equivalence, Phys Chem Chem Phys 17, 5913 (2015).

[18] B. Kharkov, X. Duan, J. W. Canary, and A. Jerschow, Effect of convection and b1 inhomogeneity on singlet relaxation experiments, J Magn Reson 284, 1 (2017).

[19] Dinesh and M. T. Rogers, Proton Spin-Lattice Relaxation in Liquid Chloroform, J Chem Phys 56, 542 (1972).

[20] H. J. Bender and M. D. Zeidler, Translational and Anisotropic Rotational Diffusion in Liquid Chloroform as Studied by NMR Relaxation, Ber Bunsenges phys Chem 75, 236 (1971).

[21] J. Cavanagh, W. J. Fairbrother, A. G. Palmer III, and N. J. Skelton, Protein NMR spectroscopy: principles and practice (Elsevier, 1995).

[22] R. E. D. McClung, Spin-Rotation Relaxation Theory, Vol. 91 (John Wiley \& Sons, Ltd, Chichester, UK, 2007).

[23] J. Kowalewski and L. Mäler, Nuclear spin relaxation in liquids: theory, experiments, and applications, 2nd ed. (CRC press, 2017).

[24] J. Kowalewski, D. Kruk, and G. Parigi, NMR relaxation in solution of paramagnetic complexes: recent theoretical progress for $\mathrm{S} \geq 1$, in Adv Inorg. Chem., Vol. 57 (Elsevier, 2005) pp. $41-104$.

[25] J. Kowalewski, L. Nordenskiöld, N. Benetis, and P.-O. Westlund, Theory of nuclear spin relaxation in paramagnetic systems in solution, Prog. Nucl. Magn. Reson. Spectrosc. 17, 141 (1985).

[26] C. L. Teng, H. Hong, S. Kiihne, and R. G. Bryant, Molecular oxygen spin-lattice relaxation in solutions measured by proton magnetic relaxation dispersion., J. Magn. Reson. 148, 31 (2001).

[27] G. Pileio and M. H. Levitt, Isotropic filtering using polyhedral phase cycles: Application to singlet state NMR, J. Magn. Reson. 191, 148 (2008).

[28] G. Pileio, Singlet state relaxation via intermolecular dipolar coupling, J. Chem. Phys. 134, 214505 (2011). 
[29] A. Moysiadi, F. Giustiniano, A. M. R. Hall, T. A. A. Cartlidge, L. J. Brown, and G. Pileio, Nuclear spin relaxation of longitudinal and singlet order in liquid-co2 solutions, Frontiers Chem. 9, 243 (2021). 


\title{
SUPPLEMENTAL MATERIAL
}

\section{Weak nuclear spin singlet relaxation mechanisms revealed by experiment and computation}

\author{
Boris Kharkov, ${ }^{1}$ Xueyou Duan, ${ }^{1}$ Jyrki Rantaharju, ${ }^{2}$ Mohamed Sabba, ${ }^{2}$ \\ Malcolm H. Levitt, ${ }^{2}$ James W. Canary, ${ }^{1}$ and Alexej Jerschow*1 \\ ${ }^{1}$ Department of Chemistry, New York University, New York, NY 10003 \\ ${ }^{2}$ School of Chemistry, University of Southampton, Southampton, UK
}




\section{NMR SPECTROSCOPY}

All NMR measurements were performed on the same degassed sample as described above to ensure the same amount of dissolved oxygen. The chloroform ${ }^{1} \mathrm{H} T_{1}$ measurements were performed on the residual $\mathrm{CHCl}_{3}$ in the $\mathrm{CDCl}_{3}$ solvent $(<0.2 \%)$. The maleate $T_{1}$ and $T_{S}$ measurements were performed on the vinylene protons of the deuterated EPM solute.

The experiments were performed on a Bruker AV500 (500 MHz, $11.74 \mathrm{~T}$ ) spectrometer with a broad-band direct observe probe. Table S1 lists the pulse sequence parameters optimized for the sequence shown in Fig. 3. The pulsed field gradients for the T00 filter were set to 35,30 , and $25 \%$ and their durations were set to 2.4, 1.6, and $1 \mathrm{~ms}$, respectively, to avoid accidental refocusing of quenched magnetization components.

TABLE S1. Pulse sequence parameters for singlet measurements. $n_{1}, n_{2}$ are the M2S loop and delay parameters corresponding to Fig. $1 . \tau_{p}$ is the $\pi / 2$ pulse duration, $n_{s}$ the number of scans, and $T_{r}$ the recycle delay.

\begin{tabular}{lrrrrrr}
$T / \mathrm{K}$ & $n_{1}$ & $n_{2}$ & $\Delta / m s$ & $\tau_{p} / \mu \mathrm{s}$ & $n_{s}$ & $T_{r} / \mathrm{s}$ \\
\hline 233.15 & 10 & 5 & 20.65 & 17.0 & 4 & 25 \\
253.15 & 23 & 11 & 20.65 & 16.9 & 4 & 35 \\
283.15 & 26 & 13 & 20.60 & 17.4 & 4 & 50 \\
298.15 & 18 & 9 & 20.25 & 16.1 & 6 & 45
\end{tabular}

The chemical shift difference between the two vinylene protons varied with temperature and the usable range was $0.5-2 \mathrm{~Hz}$. Between the temperatures 253 and $283 \mathrm{~K}$, the difference was found to be too small to efficiently generate SO. The M2S parameters were optimized for each temperature and are given in Table S1. The chemical shift differences determined from the optimized $n_{1}$ loop parameter are shown in Fig. S1 vs. temperature.

For $T_{1}$ measurements, the saturation-recovery sequence was used with a T00 filter for the saturation step.

\section{SYNTHESIS AND SAMPLE PREPARATION}

All reagents were purchased from commercial sources and used without further purification. Maleic anhydride and anhydrous ethanol- $\mathrm{d}_{6}$, were purchased from Sigma Aldrich. 


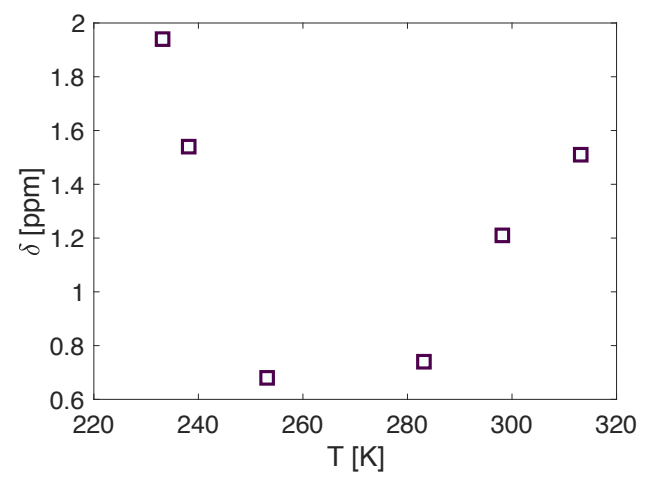

FIG. S1. Chemical shift difference between the vinylene protons, as inferred from the M2S seqeunce parameter optimization [1].

n-propyl- $\mathrm{d}_{7}$ alcohol was purchased from C/D/D isotope. Triethylamine was purchased from Fisher Scientific. All NMR solvents were purchased from Cambridge Isotopes. All compounds were analyzed by Bruker AV-500 (500 MHz, $11.74 \mathrm{~T})$. Deuterated asymmetric maleate acid esters were synthesized from maleic anhydride by following the procedure in the literature [2]. All reactions sensitive to moisture were done under inert atmosphere (Ar protection) with use of anhydrous solvents taken from a standard solvent-drying system. $\mathrm{CDCl}_{3}$ was obtained from Sigma Aldrich at $>99.8 \%$ purity. Constricted sealable NMR tubes were purchased from Norell. Samples were dissolved in deuterated chloroform and subjected to 5 cycles of freeze-pump-thaw degassing and the NMR tubes were subsequently flame-sealed.

The height of the solution in the NMR tube was approximately $9 \mathrm{~mm}$ and the tube was positioned such that it fit completely within the coil volume to avoid convection artifacts[1].

\section{COMPUTATIONAL PARAMETERS AND SETUP}

Molecular dynamics (MD) simulations were performed using Amber20. The system was prepared in antechamber using bcc charges, and the general Amber force field (GAFF) was used for parameterization. The molecule, ethyl-propyl maleate was prepared and solvated by $\mathrm{CHCl}_{3}$ in an isotropic box of $50 \AA$ size using Amber's antechamber and tleap programs. The box also contained one $\mathrm{O}_{2}$ molecule to model paramagnetic relaxation due to oxygen. Following that, the system energy was minimized using 3000 steps with the steepest 
descent method and 2000 steps with the conjugate gradient method. The system was subsequently heated to the desired temperature in 20,000 steps using a Langevin thermostat with gamma_ln=5. Stabilization at the target temperature was performed at constant temperature (gamma_ln=5) and pressure, using a Berendsen barostat with a pressure relaxation time of 1 ps, for 100,000 steps. Following that, a restart file was saved after every 50,000 steps (to give a total of 100 restart snapshots as samples of an NPT ensemble). Each of the snapshots was used for a short CPU production run of 20,000 steps using unconstrained NVE ensemble conditions. Following that, a production run of 10 million steps was performed using Amber's GPU code (pmemd.cuda) for each of the samples. A timestep of 0.2 fs and a cutoff of $11 \AA$ for electrostatic interactions were used throughout. Periodic boundary conditions were used for heating, stabilization, and production, and the SHAKE algorithm for hydrogen bonds was used for stabilization and production. Stabilization and equilibration were checked by monitoring density and temperature, which were found to remain close to equilibrium values. The use of the NPT samples was particularly important for ensuring good convergence and averaging for calculations involving oxygen. Trajectories were analyzed using the MDAnalysis package [3].

From the production run at 220K, we extracted 100 random conformations of the molecule, and performed a geometry optimization using using the B3LYP DFT functional, a 6-31G(d) basis set, and implicit chloroform solvent using Gaussian 16 software to find the local minimum. Convergence was checked via a frequency calculation. From these converged structures, we calculated CSA tensors using the same functional and basis set. The CSA tensors were separated into their traceless symmetric and antisymmetric components. For the relaxation expressions, two types of averages of the tensor norms were calculated: (1) individual tensor norm averages for spin-lattice relaxation rate constant $\left(R_{1}\right)$ calculations, and (2) the averages of the norms of the differences of the tensors of the two phosphate nuclei for SO relaxation rate constant $\left(R_{S}\right)$ calculations.

500 random starting points were chosen from trajectories and the results averaged. Each correlation function was calculated over 4000 points (800 ps).

To improve statistics, the correlation functions for spin-pairs were averaged for calculations obtained from switching the $x, y$, and $z$ axes when calculating the $P_{2}$ function values. 


\section{Evaluation of the fast motion regime assumption}

The largest intermolecular contribution was found to be due to coupling to $\mathrm{Cl}$ spins of the solvent, and the slowest motion was found for the lowest examined temperature (220K). For this situation, the correlation function is shown in Fig. S2. This curve can be fit very well with a biexponential function to give two correlation times, 77.3 ps and 11.1 ps. From these two correlation times one may determine the deviation from the fast motion assumption as being at the levels of $9.6 \cdot 10^{-4}$ and $1.96 \cdot 10^{-5}$, both of which can be neglected. Therefore the use of the fast motion approximation is well justified.



FIG. S2. Correlation function for the intermolecular ${ }^{1} \mathrm{H}-{ }^{35,37} \mathrm{Cl}$ dipolar coupling contribution to $R_{1}$ of the vinylene $1 \mathrm{H}$ of the EPM molecule, along with a biexponential fit.

\section{Convergence of relaxation calculations}

In order to determine a balance between computational cost and accuracy, we analyzed the convergence behavior of the intermolecular relaxation expressions for $R_{S}$ as a function of the cutoff distance used to determine the coupling partners. Fig. S3 shows that a cutoff distance of $20 \AA$ can be considered as producing well converged results. The difference in value between $20 \AA$ and $31 \AA$ cutoff is below $1 \%$.

\section{DERIVATION OF THE SPIN-ROTATION RELAXATION EXPRESSION}

The Hubbard model spin-rotation contribution to the $R_{1}$ relaxation rate constant for non-spherical molecules is given in Eq. (22) of Ref. [4]. For the axially symmetric case, 


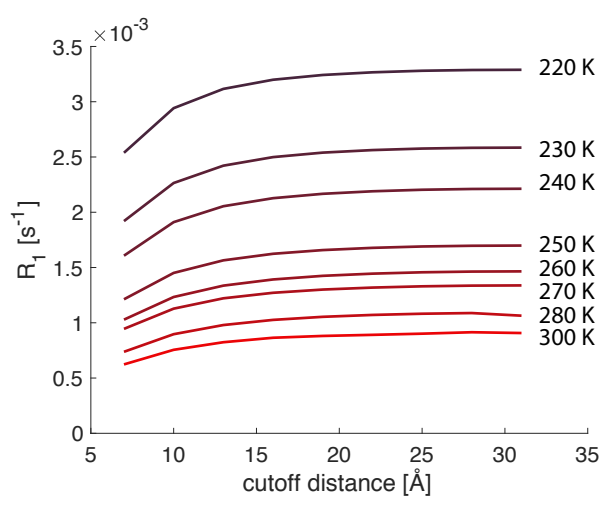

FIG. S3. $R_{S}$ relaxation rate constants for the intermolecular ${ }^{1} \mathrm{H}-{ }^{35,37} \mathrm{Cl}$ contribution as a function of cutoff distance.

expressed with the parallel and perpendicular friction constants $\epsilon_{\|}$and $\epsilon_{\perp}$, respectively, the equation takes the form (see Eq. (18) of Ref. [4])

$$
R_{1}^{s r}=\frac{2 k_{B} T}{3 \hbar^{2}}\left(I_{\|}^{2} C_{\|}^{2} / \epsilon_{\|}+2 I_{\perp}^{2} C_{\perp}^{2} / \epsilon_{\perp}\right)
$$

where $I_{\|}$and $I_{\perp}$ are the moments of inertia for rotation around the $\mathrm{C}-\mathrm{H}$ bond, and perpendicular to it, respectively. The friction constants and diffusion constants $\mathrm{D}_{\|, \perp}$ have the relationship (see Eq. (6.39) in Kowalewski and Mäler [5])

$$
\epsilon_{\|, \perp}=\frac{k_{B} T}{\mathrm{D}_{\|, \perp}} .
$$

With these relationships, one obtains for the spin-rotation contribution

$$
R_{1}^{s r}=\frac{2}{3 \hbar^{2}}\left(I_{\|}^{2} C_{\|}^{2} \mathrm{D}_{\|}+2 I_{\perp}^{2} C_{\perp}^{2} \mathrm{D}_{\perp}\right)
$$

\section{CALCULATION OF CSA CONTRIBUTIONS TO $R_{1}$ AND $R_{S}$}

The expressions for the $R_{1}$ component calculations are

$$
\begin{gathered}
R_{1}^{\text {sym }}=\frac{2}{15}\left(\omega_{0} \sqrt{3 / 2}\left\|\sigma_{\text {sym }}\right\|_{F}\right)^{2} \frac{\tau_{2}}{1+\left(\omega_{0} \tau_{2}\right)^{2}} \\
R_{1}^{\text {anti }}=\frac{1}{6}\left(\omega_{0}\left\|\sigma_{\text {anti }}\right\|_{F}\right)^{2} \frac{\tau_{1}}{1+\left(\omega_{0} \tau_{1}\right)^{2}} .
\end{gathered}
$$

Here, $\sigma_{\text {sym }}$ and $\sigma_{\text {anti }}$ designate the traceless symmetric and the antisymmetric tensor components, respectively, and $\|\sigma\|_{F}$ indicates the Frobenius norm of a tensor, i.e. the square 
root of the sum over the squares of all tensor elements. $\tau_{2}$ is the second rank correlation time. For isotropic motion, one may assume that the first rank correlation time $\tau_{1}=3 \tau_{2}$. In the context of singlet-state relaxation, it was shown specifically that the antisymmetric component could become a major relaxation contribution [6]. In that work, the expressions for the $R_{S}$ components were provided in the fast motion regime. Outside of that regime, the expressions become

$$
\begin{gathered}
R_{S}^{\text {sym }}=\frac{2}{9}\left(\omega_{0}\left\|\Delta \sigma_{\text {sym }}\right\|_{F}\right)^{2} \frac{1}{5}\left(2 \tau_{2}+\frac{3 \tau_{2}}{1+\left(\omega_{0} \tau_{2}\right)^{2}}\right) \\
R_{S}^{\text {anti }}=\frac{2}{9}\left(\omega_{0}\left\|\Delta \sigma_{\text {anti }}\right\|_{F}\right)^{2} \frac{\tau_{1}}{1+\left(\omega_{0} \tau_{1}\right)^{2}},
\end{gathered}
$$

where $\Delta \sigma_{\text {sym }}$ and $\Delta \sigma_{\text {anti }}$ represent the symmetric and the antisymmetric components of the difference between the tensors at the two phosphorus sites. For the $R_{1}$ calculations, the correlation time of the $\mathrm{C}-\mathrm{H}$ bond vector reorientation was extracted from the MD trajectories. For the $R_{S}$ calculations, the correlation time of the ${ }^{1} \mathrm{H}-{ }^{1} \mathrm{H}$ vinylene internuclear vector reorientation was used. These correlation times were obtained by calculating

$$
a_{1}(t)=\cos (\theta(t))
$$

with the subscript 1 indicating the rank, and $\theta$ being the angle of the vector with respect to the $z$-axis. The $k$-th rank correlation function was calculated by

$$
C_{k}(t)=\overline{a_{k}\left(t^{\prime}\right)^{*} a_{k}\left(t+t^{\prime}\right)}
$$

where the average was performed over $t^{\prime}$, and the correlation time was calculated by

$$
\tau_{k}=\int_{0}^{t_{\max }} C_{k}(t) / C_{k}(0)
$$

The sum was performed over $t_{\max }=800 \mathrm{ps}$.

From this result, the second-rank correlation time was calculated using $\tau_{2}=\tau_{1} / 3$ and used for the symmetric CSA interaction.

Fig. S4 shows the tensor norms for the conformations extracted from the MD trajectory. The CSA difference tensor norms were found to be $\left\|\Delta \sigma_{\text {sym }}\right\|_{F}=4.35 \mathrm{ppm}$ and $\left\|\Delta \sigma_{\text {anti }}\right\|_{F}=$ $0.94 \mathrm{ppm}$. The standard deviations were 0.13 and $0.18 \mathrm{ppm}$, respectively.

Fig. 4 shows representative snapshots with a visualization of both the symmetric and the antisymmetric components of the CSA tensors. The symmetric CSA tensors were visualized 

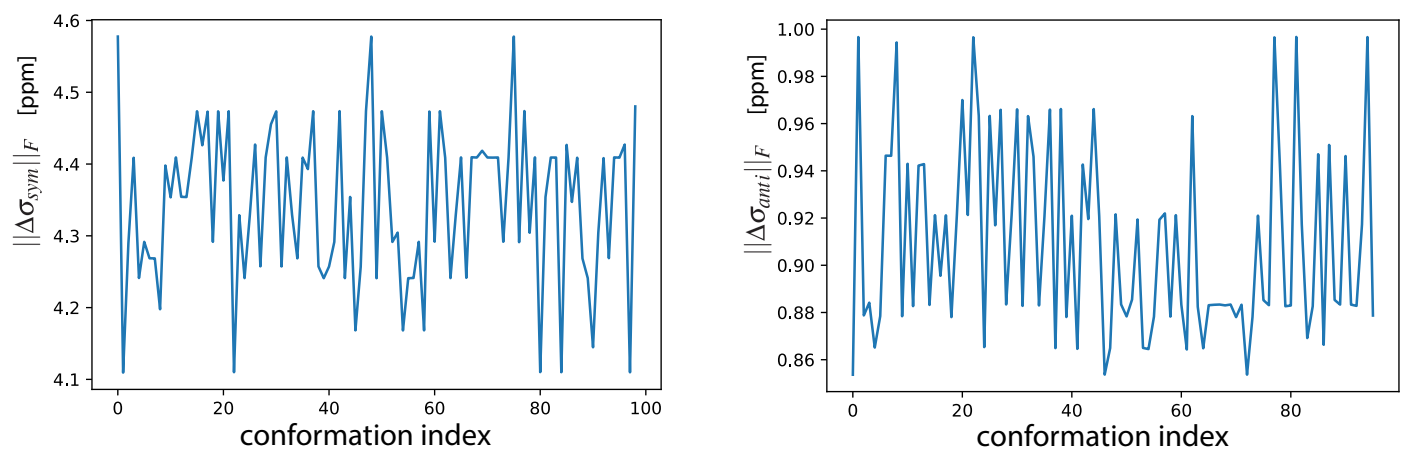

FIG. S4. Frobenius norms of the symmetric and antisymmetric components of the CSA difference tensors for the conformations extracted from the MD trajectory.

with SpinDynamica v. 3.6 [7] using the function Ovaloid (which was based on the procedure described previously $[8,9]$ ), and molecular graphics were created using Mathematica's Molecule visualization function. The antisymmetric tensor was visualized as follows:[10, 11] The antisymmetric tensor $\sigma_{\text {anti }}$ can be written as

$$
\left[\begin{array}{ccc}
0 & -z & y \\
z & 0 & x \\
-y & -x & 0
\end{array}\right]
$$

which can be recast in vector form $\mathbf{v}=(x, y, z)$ and represented by the arrows shown in Fig. 4b. The antisymmetric shielding tensors are found to vanish if the $\mathrm{H}-\mathrm{C}-\mathrm{C}-\mathrm{H}$ moiety is in a single plane.

\section{PARAMAGNETIC RELAXATION}

The relaxation of nuclei by the interaction with unpaired electrons is a complex topic[12, 13]. A detailed treatment of this problem requires good understanding of the electron spin interactions including zero-field splittings. For the sake of simplicity and tractability, we employed a simplified treatment based on Solomon relaxation of the $\mathrm{O}_{2}$ electron spin pair. The relaxation contribution was determined as follows:

$$
R_{1, S}^{O_{2}}=R_{1, S}^{O_{2}, d d}+\frac{1}{T_{1 e}},
$$

where $R_{1, S}^{O_{2}, d d}$ is the spin-lattice relaxation or the $\mathrm{SO}$ order relaxation rate constant determined using the procedure above for intermolecular dipolar relaxation contributions, with 


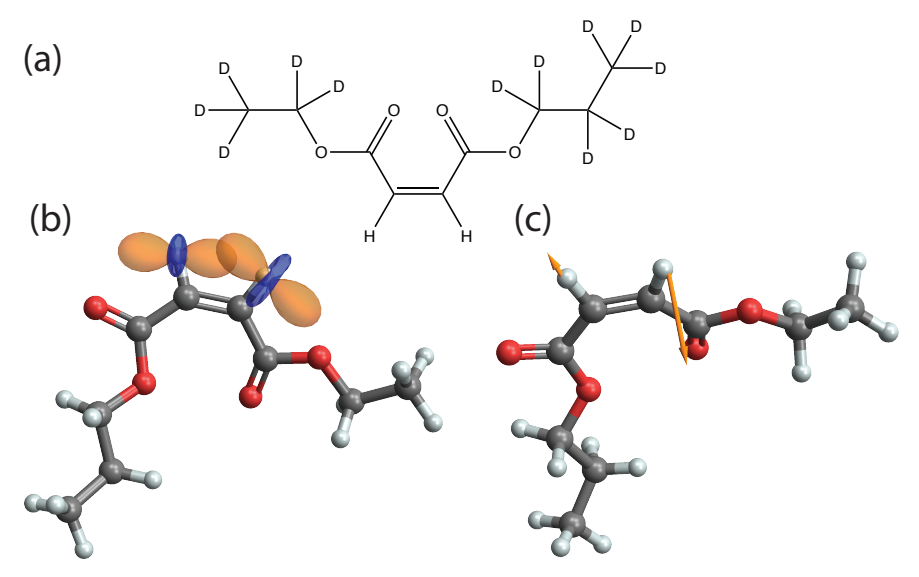

FIG. S5. (a) Ethyl $-\mathrm{d}_{5}$ propyl $-\mathrm{d}_{7}$ maleate molecule. The singlet was prepared for the vinylene protons in the center. (b) Representation of the symmetric CSA tensor components as ovaloid surfaces centred at the vinylene proton positions, and (c) representation of the antisymmetric CSA tensor components by arrows originating from the vinylene proton positions.

$S=1$ for the unpaired electrons of $\mathrm{O}_{2}$ and the gyromagnetic ratio for the electron. For the electron relaxation time $T_{1 e}$, we used the reported the average value of $7.5 \mathrm{ps}$ [14]. The influence of $T_{1 e}$ on $R_{1}$ relaxation rate constants is shown in Fig. S3. The paramagnetic interaction was then scaled to fit the total $R_{1}$ rate to the experimental values. The same scaling factor was subsequently used for the calculation of the paramagnetic contribution to $R_{S}$.

As a result, we obtain a self-consistent procedure for determining the paramagnetic relaxation contribution.

A distance cutoff of $20 \AA$ was used. The effective oxygen concentration needed to be evaluated over the volume $\left(\frac{4}{3} \pi r^{3}\right)$, with $r$ being the cutoff distance. When evaluated over the simulation box, the effective oxygen concentration was approximately $12 \mathrm{mM}$.

\section{VALIDATION OF COMPUTED $R_{1}$ RATES FOR EPM MOLECULE}

Fig. S4 shows the experimental values for the $R_{1}$ rate constants determined for the vinylene protons of the EPM compound, along with the values obtained from MD simulations. No distinction between the rate constants for the two spins could be observed. The dominating mechanism for vinylene $T_{1}$ is the intra-pair dipolar coupling. Other mechanisms can be shown to be at least two orders of magnitude smaller. Other mechanisms such as 


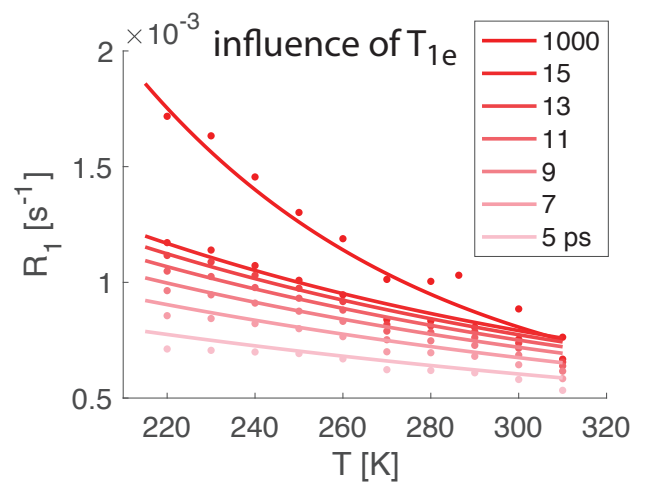

FIG. S6. The influence of $T_{1 e}$ on the contribution of oxygen to chloroform $R_{1}$.

intra-molecular and intermolecular dipolar couplings, CSA relaxation, and the contribution of oxygen below, can be shown to range from $1 \times 10^{-3} \mathrm{~s}^{-1}$ to $5 \times 10^{-3} \mathrm{~s}^{-1}$, and are therefore approximately two orders of magnitude lower, and can hence be ignored in this calculation. As is seen in Fig. S4, the agreement between experiment and calculation is very good, providing confidence in the procedure. Furthermore, because the dominating mechanism has very few parameters (e.g. the distance between the protons is largely fixed), the observed correspondence between calculation and experiment also indicates that the reorientation correlation times calculated from MD trajectories must be fairly accurate over the observed temperature range.

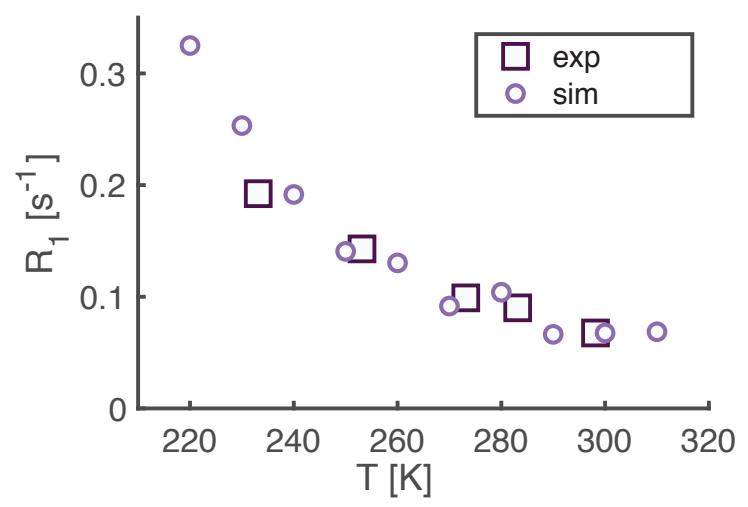

FIG. S7. Experimental (exp) and calculated $(\operatorname{sim}) R_{1}$ rate constants for the vinylene protons in EPM. 
[1] B. Kharkov, X. Duan, J. W. Canary, and A. Jerschow, Effect of convection and b1 inhomogeneity on singlet relaxation experiments, J Magn Reson 284, 1 (2017).

[2] G. Stevanato, S. S. Roy, J. Hill-Cousins, I. Kuprov, L. J. Brown, R. C. Brown, G. Pileio, and M. H. Levitt, Long-lived nuclear spin states far from magnetic equivalence, Phys Chem Chem Phys 17, 5913 (2015).

[3] N. Michaud-Agrawal, E. J. Denning, T. B. Woolf, and O. Beckstein, Mdanalysis: a toolkit for the analysis of molecular dynamics simulations, J Comput Chem 32, 2319 (2011).

[4] R. E. D. McClung, Spin-Rotation Relaxation Theory, Vol. 91 (John Wiley \& Sons, Ltd, Chichester, UK, 2007).

[5] J. Kowalewski and L. Mäler, Nuclear spin relaxation in liquids: theory, experiments, and applications, 2nd ed. (CRC press, 2017).

[6] G. Pileio, J. T. Hill-Cousins, S. Mitchell, I. Kuprov, L. J. Brown, R. C. Brown, and M. H. Levitt, Long-lived nuclear singlet order in near-equivalent 13c spin pairs, J Am Chem Soc 134, 17494 (2012).

[7] C. Bengs and M. H. Levitt, Spindynamica: Symbolic and numerical magnetic resonance in a mathematica environment, Magn Reson Chem 56, 374 (2018).

[8] R. Radeglia, On the pictorial representation of the magnetic screening tensor: ellipsoid or ovaloid?, Solid State Nucl Magn Reson 4, 317 (1995).

[9] R. P. Young, C. R. Lewis, C. Yang, L. Wang, J. K. Harper, and L. J. Mueller, Tensorview: A software tool for displaying nmr tensors, Magn Reson Chem 57, 211 (2019).

[10] A. Buckingham and S. Malm, Asymmetry in the nuclear magnetic shielding tensor, Mol. Phys. 22, 1127 (1971), https://doi.org/10.1080/00268977100103431.

[11] F. A. L. Anet and D. J. O'Leary, The shielding tensor. Part I: Understanding its symmetry properties, Concepts Magn. Reson. 3, 193 (1991).

[12] J. Kowalewski, D. Kruk, and G. Parigi, NMR relaxation in solution of paramagnetic complexes: recent theoretical progress for $\mathrm{S} \geq 1$, in Adv Inorg. Chem., Vol. 57 (Elsevier, 2005) pp. $41-104$.

[13] J. Kowalewski, L. Nordenskiöld, N. Benetis, and P.-O. Westlund, Theory of nuclear spin relaxation in paramagnetic systems in solution, Prog. Nucl. Magn. Reson. Spectrosc. 17, 141 
(1985).

[14] C. L. Teng, H. Hong, S. Kiihne, and R. G. Bryant, Molecular oxygen spin-lattice relaxation in solutions measured by proton magnetic relaxation dispersion., J. Magn. Reson. 148, 31 (2001). 